\title{
The effect of acetic acid extraction time on the dissolution of lead and cadmium in ceramic tiles
}

\author{
Tao Tian*, Yujing Zhang ${ }^{1}$, Zhiyuan Kang ${ }^{1}$, Haibo Dang ${ }^{1}$ \\ ${ }^{1}$ China Building Material Test \& Certification Group(Shaanxi) Co. Ltd, Xi'an, Shaanxi, China, 710049 \\ *China Building Material Test \& Certification Group(Shaanxi) Co. Ltd, Xi'an, Shaanxi, China, 710049
}

\begin{abstract}
With the improvement of people's living standard, the glazed tiles will be decorated with some colored glaze materials in order to increase strength and surface decoration effect, and these ceramic tiles containing lead and cadmium will be dissolved out under the action of acid. The dissolved lead and cadmium and other heavy metals after inhalation will affect the human liver, nervous system, hematopoiesis, and even paralysis. The Influence of detection mechanism and detection time on the results is discussed in this paper.
\end{abstract}

\section{Reasons for the introduction of lead and cadmium into ceramic tiles}

The addition of lead compounds, such as lead lead lead, can play a role in fusing, reducing surface tension, increasing the gloss of glaze, and effectively promote pigment coloring at a lower temperature. Compounds added with cadmium, such as cadmium carbonate, are mainly used as colorants, which can make ceramic tiles have a bright and bright color appearance at high temperature. The cadmium oxide in the glaze can improve the stability of the pigment, so that it can still maintain the color stability of the brick surface under the interference of strong oxidants.

\section{2 experiment}

\section{1 The experimental principle}

There is a chemical reaction between the ceramic brick and the acid solution. In the reaction process, the free glass will dissolve from the surface of the glass to the acid solution due to the role of the hydrated hydrogen ion in the acid solution. These free glass ions first enter the holes in the network structure sequentially, and then the acid solution separates alkali ions and lead ions from these holes. Because the bond between base ion and oxygen is weaker than that between lead ion and oxygen,alkali ions in glass are more likely to be separated from lead by acetic acid solution than lead ions, leaving many vacancies in the glass structure, which are occupied by lead ions diffusing into the surface. Then the lead ions accumulate on the surface of the glass where the reaction occurs, and are finally separated by the acid solution. The leaching mechanism of cadmium from glaze and glass by acid solution is similar to that of lead.

\section{2 The experimental method}

(1) Prepare 3 bricks to be tested, clean the surface to be tested, remove the debris on the surface of the brick that may affect the results of the experiment, rinse it with secondary distilled water, and dry it with a soft cleaning cloth for use.

(2) Surround the brick surface with a circle of $6 \mathrm{~mm}$ high and $4 \mathrm{~mm}$ wide silicone sealant. The strip of silicone sealant should be intact, and the silicone sealant should be tightly combined with the brick surface, and the sealant should be dried overnight.

(3) Prepare 4\% acetic acid solution for use, fill the ceramic tile surface sealed with silicone sealant with $4 \%$ acetic acid solution, and record the volume $\mathrm{V}$ of the added acetic acid solution. Place it in a room with a temperature of $22+2^{\circ} \mathrm{C}$ and cover it with an impermeable cover to prevent the solution from being contaminated or evaporated.

(4) After standing for 24 hours, the solution was collected and measured by atomic absorption spectrophotometry.

\section{Conclusion}

This paper studies the effect of acetic acid storage time on the results of lead and cadmium dissolution during pretreatment

\section{1 sample}

We chose two kinds of glazed flat bricks and made samples for experiment. 


\section{2 Experimental process}

The two groups of samples were subjected to the same pre-treatment process to ensure the same experimental environment, sample size and added volume of acetic acid solution. The solution to be tested was extracted at $12 \mathrm{~h}, 16 \mathrm{~h}, 20 \mathrm{~h}, 24 \mathrm{~h}, 28 \mathrm{~h}$ and $32 \mathrm{~h}$ respectively to determine the lead and cadmium dissolution concentration.

\section{3 experimental result}

The experimental results are as follows:

Table $1 \mathrm{~Pb}$ concentration of ceramic tiles under different treatment time

\begin{tabular}{|l|l|l|l|l|l|l|}
\hline \multirow{2}{*}{ Sample } & \multicolumn{6}{l}{ Pb concentration under different treatment time (mg/L) } \\
\cline { 2 - 7 } & $12 \mathrm{~h}$ & $16 \mathrm{~h}$ & $20 \mathrm{~h}$ & $24 \mathrm{~h}$ & $28 \mathrm{~h}$ & $32 \mathrm{~h}$ \\
\hline Sample 1 & 0.192 & 0.210 & 0.247 & 0.270 & 0.271 & 0.271 \\
\hline Sample 2 & 0.177 & 0.196 & 0.217 & 0.231 & 0.231 & 0.231 \\
\hline
\end{tabular}

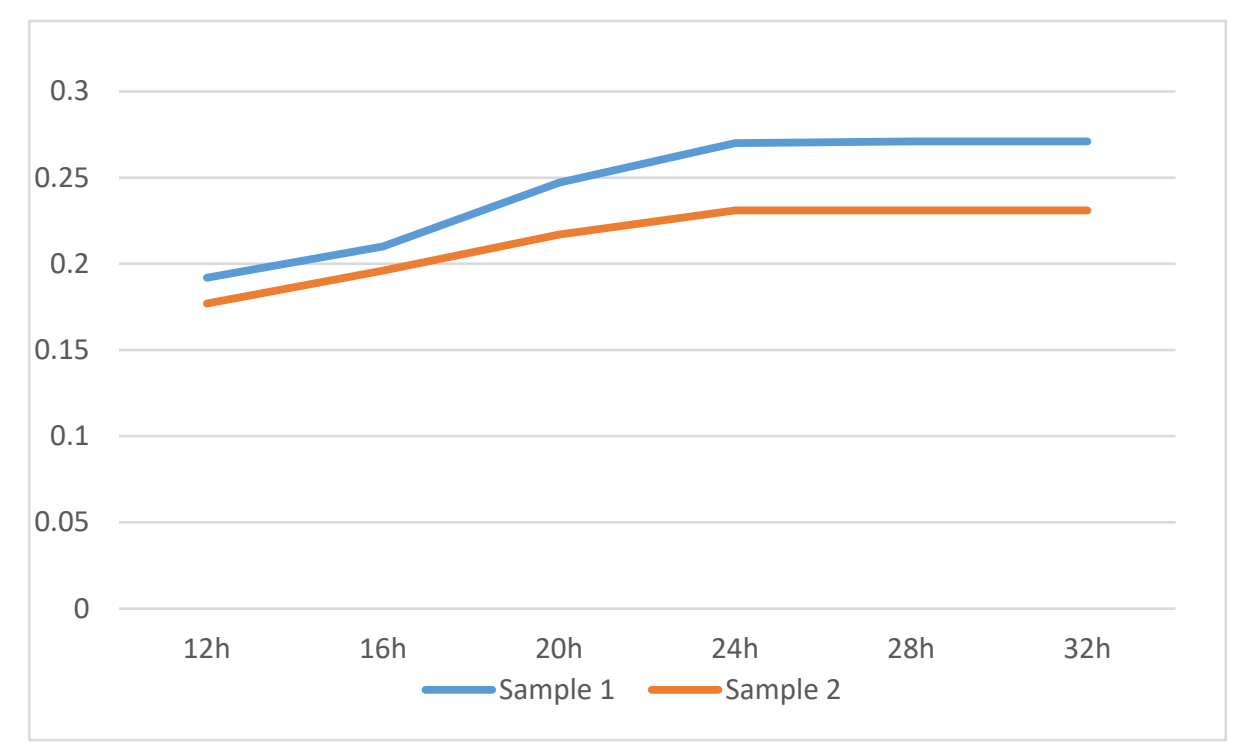

FIG. $1 \mathrm{~Pb}$ concentrations of ceramic tiles under different treatment times

Table $2 \mathrm{Cd}$ concentration of ceramic tiles under different treatment time

\begin{tabular}{|l|l|l|l|l|l|l|}
\hline \multirow{2}{*}{ Sample } & \multicolumn{6}{|l|}{ Cd concentrations at different treatment times (mg/L) } \\
\cline { 2 - 7 } & $12 \mathrm{~h}$ & $16 \mathrm{~h}$ & $20 \mathrm{~h}$ & $24 \mathrm{~h}$ & $28 \mathrm{~h}$ & $32 \mathrm{~h}$ \\
\hline Sample 1 & 0.012 & 0.012 & 0.014 & 0.017 & 0.019 & 0.017 \\
\hline Sample 2 & 0.013 & 0.019 & 0.020 & 0.026 & 0.026 & 0.026 \\
\hline
\end{tabular}




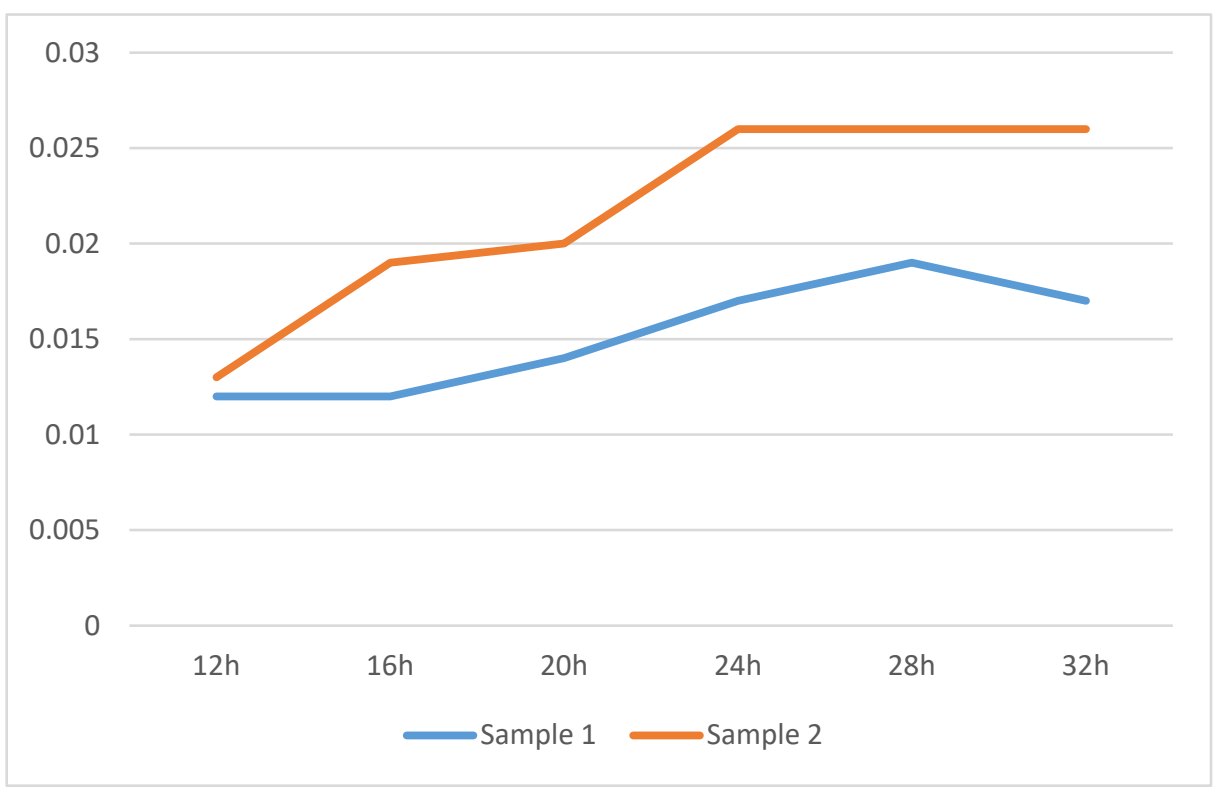

FIG. $2 \mathrm{Cd}$ concentration of ceramic tiles under different treatment times

Under the same condition of other pretreatment conditions, the concentration of $\mathrm{Pb}$ and $\mathrm{CD}$ changed with the time of acetic acid extraction. The concentration of $\mathrm{Pb}$ and $\mathrm{Cd}$ increased with the time of acetic acid extraction, reaching the maximum at $24 \mathrm{~h}$, and then kept a constant state.

\section{4. analysis}

Acetic acid extraction time is an important factor in the detection process of the lead and cadmium dissolution of glazed bricks. When the extraction time is short, the dissolution of lead and cadmium is not complete. When the extraction time exceeds $24 \mathrm{~h}$, the dissolution of lead and cadmium reaches saturation. Considering the time cost and working time, it is most appropriate to select the acetic acid extraction time as $24 \mathrm{~h}$.

\section{Reference}

1. $\mathrm{J} \mathrm{Hu}, \mathrm{Z} \mathrm{Qu}$. Causes, hazards and detection of lead and cadmium dissolution from domestic ceramics $[\mathrm{J}]$. Foshan ceramics, 21 (11): 16-19 (2011)

2. D Wu,L Wang, L Lv.Determination of Lead and Cadmium Dissolution in Ceramics for Daily Use[J].Guangzhou Chemical Industry,2011,39(10):127-129.

3. J Jia,W Dong, N Xu.Research and Application of Spectrophotometric Method for Lead Content in Ceramics[J].Journal of Tangshan Normal University,2006(02):45-47.

4. W Li , Y Dong, H Wang.Study on the influence of sample pretreatment methods on the determination of lead and cadmium dissolution in daily ceramics $[\mathrm{J}]$.China Ceramic Industry,2016,23(04):11-18.
5. What are the hazards to the human body caused by excessive lead and cadmium content in daily ceramics $[\mathrm{J}]$.Supervision Selection,2004(10):59. 